Archives de sciences sociales des religions L'héritage des Formes

\title{
Durkheim et le problème de l'étude sociologique de la religion
}

Joseph Sumpf

\section{(2) OpenEdition \\ Journals}

Édition électronique

URL : http://journals.openedition.org/assr/23023

ISSN : 1777-5825

Éditeur

Éditions de l'EHESS

Référence électronique

Joseph Sumpf, «Durkheim et le problème de l'étude sociologique de la religion », Archives de sciences sociales des religions [En ligne], L'héritage des Formes, mis en ligne le 18 juillet 2011, consulté le 20 avril 2019. URL : http://journals.openedition.org/assr/23023 


\section{Durkheim et le problème de l'étude sociologique de la religion .}

In: Archives des sciences sociales des religions. N. 20, 1965. pp. 63-73.

Citer ce document / Cite this document :

Sumpf Joseph. Durkheim et le problème de l'étude sociologique de la religion. In: Archives des sciences sociales des religions. N. 20, 1965. pp. 63-73.

doi : 10.3406/assr.1965.1786

http://www.persee.fr/web/revues/home/prescript/article/assr_0003-9659_1965_num_20_1_1786 


\title{
DURKHEIM ET LE PROBLÈME DE L'ÉTUDE SOCIOLOGIQUE DE LA RELIGION
}

\begin{abstract}
$\mathrm{D}$
URKheim, dans une lettre adressée à la Revue Néo-Scolastique de Louvain, réfutant un certain nombre d'allégations sur la genèse de sa pensée, écrit : "C'est seulement en 1895 que j'eus le sentiment net du rôle capital joué par The religion dans la vie sociale. C'est en cette année que pour la première fois, je Yrouvai le moyen d'aborder sociologiquement l'étude de la religion. Ce fut pour moi une révélation. Ce cours de 1895 marque une ligne de démarcation dans le Uléveloppement de ma pensée si bien que toutes mes recherches antérieures Carent être reprises à nouveaux frais pour être mises en harmonie avec ces vues nosuvelles" (1). A défaut du cours de 1895 , tentons d'expliquer le sens de ce Sexte.

En premier lieu, nous apprenons que Durkheim a trouvé un moyen d'aborder sociologiquement l'étude de la religion. Qu'est-ce à dire ? En 1886, rendant compte â l'ouvrage de Spencer Ecclesiastical Institutions (2), il assimile en un sens le droit, la morale et la religion. Car il s'agit dans les trois cas de systèmes régulateurs de la vie sociale. Dieu n'est que le symbole relativement accidentel de l'institution.

Cependant "ce qui, peut-être, distingue le mieux cette forme de toutes les autres, c'est qu'elle s'impose non seulement à la conduite mais à la conscience. Elle ne dicte pas seulement des actes mais des idées et des sentiments. En définitive, la religion commence avec la foi " (3).

D'autre part la religion n'est pas une philosophie vague à l'usage du peuple comme le veut Spencer. Elle ne meurt pas avec l'avènement de la raison. Elle est dans le corps même de la vie sociale : "Tant qu'il y aura des hommes, il y aura entre eux quelque foi commune" (4).
\end{abstract}

(1) Simon Deploige, Le Conflit de la morale et de la sociologie, Louvain, Institut supérieur de philosophie, 1911, 424 pages. Deploige accuse Durkheim d'être un penseur allemand. Le texte cité fait partie d'une lettre p. 402.

(2) "Les études récentes de sciences sociales *, Revue philosophique, 11e année, juilletdécembre 1886.

(3) Ibid., p. 68.

(4) Ibid., p. 69. 


\section{ARCHIVES DE SOCIOLOGIE DES RELIGIONS}

La foi est donc l'essentiel de la religion. Ce n'est pas un simple sentiment, une aspiration vague vers l'infini. Tout en étant intime, c'est une force collective d'adhésion, mais aussi une force d'invention et d'action dans une période de crise. C'est ce qu'indiquent les cours transcrits dans $L$ 'Education Morale (cours de 1902, mais qui ne fait que reprendre des cours antérieurs). "Quand, au contraire, la morale est à constituer, quand elle se cherche, il faut bien, pour la faire, recourir non aux forces purement conservatrices, puisqu'il ne s'agit pas de conserver, mais aux forces actives et inventives de la conscience... dans les conditions présentes, c'est surtout la foi dans un commun idéal qu'il faut chercher à éveiller" (5).

La religion est donc à la fois règle et exaltation. Ce dernier trait lui est spécifique et se révèle particulièrement lorsque la société est en train de changer.

\section{I. - LFs obstacles a L'Étude SOCIOlogiQue de LA RELigion.}

Fn quel sens l'étude de la foi est-elle difficile sinon impossible pour le sociologue Durkheim, tel qu'il s'affirme dans les ouvrages antérieurs à 1897, c'est-àdire la thèse sur la Division du Travail, Les Règles, Le Suicide?

D'une part il récuse l'histoire des religions, qu'elle soit anglaise ou allemande, parce qu'elle procède par grandes généralisations (6); il récuse aussi l'ethnographie parce qu'elle sombre dans l'exotisme. D'autre part, à l'intérieur de la théorie et de la méthode durkheimiennes, les obstacles ne sont pas moindres. L'on sait qu'il s'agit de considérer les phénomènes sociaux comme des choses, de les classer puis de constituer des types. Ces traits de la sociologie durkheimienne se révèlent très complexes dès qu'on les rapproche de leur origine essentielle, la pensée de Renouvier, ou dès qu'on cherche à les penser dans la pratique actuelle de la recherche, comme le font Selvin, Lazarsfeld ou Boudon. En gros, sans entrer dans un débat qui exigerait un tout autre article, la "chose " n'est pas immédiatement donnée. Il ne s'agit ni du phénomène élaboré tel que le conçoit le positivisme, ni du fait présent à la conscience tel que l'accepte l'empirisme, ni de substances, car la substance (sociale ou non) fait problème pour Durkheim comme pour Renouvier. "Est chose tout ce qui est donné, tout ce qui s'offre ou plutôt s'impose à l'observation ", dit un passage des Règles. Ce qui s'impose c'est un ensemble de phénomènes, de représentations, dirait Renouvier. Mais cet ensemble ne va pas de soi. Le passage des représentations partielles à l'ensemble, des parties au tout, à la chose, est proprement contradictoire (7). Cela ne signifie pas pour autant que le sujet se trouve devant une poussière de sensations. Les représentations constituent des "touts" relativement constitués. Ainsi, des groupes de gens prient. Mais, ces évidences, ces genres spontanés, le sociologue, comme tout savant, les refuse. Ce sont des idoles issues de nos besoins et de notre pouvoir technique. La vraie science exige que l'évidence soit le fruit de la constitution des types.

Les faits religieux par nature se manifestent en représentations très diverses, comme en témoigne l'ethnographie, en représentations fluentes puisqu'il y a une histoire des religions, puisque la foi est un phénomène de crise de la société, en représentations mystérieuses puisque la foi est à l'intérieur de la conscience.

(5) L'Education morale, nouvelle édition, P.U.F., 1963, p. 86-87.

(6) Reoue philosophique, 1886. Article cité, "La Sociologie et l'histoire des religions sont et devraient rester choses distinctes $n$, p. 66.

(7) Durkheim attribue "surtout à Renouvier " la distinction du tout et des parties. Voir : "La sociologic en France au 19 e siècle ", Revue bleue, 10 et 26 mai 1900. 
Le sociologue Durkheim ne peut ni s'en tenir à ces représentations, encore qu'il les perçoive, ni passer à un genre comme lorsqu'il s'agit du socialisme, ni utiliser la méthode élaborée au contact du problème moral ou politique. En effet, du socialisme je puis faire un genre (8), car il s'agit "d'un cri de douleur collectif », d'un mouvement vivant, manifeste. Les mots par lesquels s'exprime le socialisme indiquent la société moderne même. C'est pourquoi "l'essentiel c'est que nous ayons devant nous un ordre de faits un et nettement circonscrit et auquel on puisse donner le nom de socialisme, sans pour cela faire violence à la langue "(9).

Il n'en est pas de même de la multitude des religions ni du christianisme. Le christianisme manifeste une hiérarchie à structure monarchique rigide, si bien que l'entreprise moderniste est illusoire (10). Il a engendré un "état de stagnation morale»(11). Je puis parler d'une Eglise. Puis-je parler d'un christianisme ? Ce n'est pas un mouvement. Ce ne peut être un genre.

Je ne puis davantage constituer des espèces ou des types. En effet, cette constitution, à suivre la méthodologie des Règles, du Suicide, implique d'abord un certain tri des phénomènes qui me donne une définition (par exemple : la définition des faits de droit : tout crime comporte un tribunal). C'est-à-dire une classe, nominale, en ce sens qu'elle est provisoire, grossière parce que relativement immédiate, réelle cependant en ce sens qu'au-delà de la classe des faits de droit, de suicide, je passe à ces «touts 》 que sont les espèces ou types. Le passage au type implique soit une sommation des parties (le tout est un agrégat - exemple : les sociétés polysegmentaires), soit un saut jusqu'à l'hypothèse, la cause (tel type de suicide). Ce dernier passage peut emprunter la voie d'une discussion dialectique au sens hamelinien du terme, c'est-à-dire corrélation, appel d'un terme par un terme contraire et non opposition des propositions contradictoires, pour aboutir à un tout, une synthèse qui obéisse au principe de convenance, c'est-à-dire manifeste une finalité interne. Il en est ainsi du droit. Les types, les espèces sont la réalité même révélée dans le fonctionnement de certaines causalités. Il en est ainsi du suicide. Mais il en est ainsi aussi de la France, espèce sociale (12), de la société moderne, etc... Il s'agit en chaque cas de l'état d'un tout social particulier donné (13) compris dans sa fonction, c'est-à-dire dans l'accomplissement (la "convenance ") normal d'une causalité (exemple : la sauvegarde sociale par le droit). Une telle théorie de la réalité sociale fait évidemment primer les circumfusa sur les praeterita, comme le marque le Doyen Davy (14). Elle se refuse à être tributaire de l'histoire. "S'il y a des espèces sociales, dit Durkheim, c'est que la vie collective dépend avant tout de conditions concomitantes qui présentent une certaine diversité. Si, au contraire, les principales causes des événements sociaux étaient toutes dans le passé, chaque peuple ne serait plus que le prolongement de celui qui l'a précédé et les différentes sociétés perdraient

(8) "C'est le genre que nous définissons", Cours sur le socialisme (1895-1896), édité par M. Mauss. Félix Alcan, 1928, 352 pages, p. 35.

(9) Ibid., p. 20.

(10) Libres entretiens, I.

(11) "L'élite intellectuclle et la démocratie ", Revue bleue, 4 juin 1904. "Certes je suis loin de penser que l'anticléricalisme suffise à tout, j'ai même hâte de voir la société s'attacher à des fins plus objectives. Mais l'essentiel était de ne pas nous laisser retomber dans l'état de stagnation morale où nous nous nommes trop longtemps attardés".

(12) Règles, P.U.F., 1947, 149 pages. Voir la note p. 88.

(13) "Ce qui existe, ce qui seul est donné à l'observation, ce sont des espèces particulières qui naissent, se développent, meurent indépendamment les unes des autres ". (Ibid., p. 20).

(14) Règles, p. 119. Cité par Georges DAvy, "Emile Durkheim ", Revue de métaphysique et morale, 1920 , p. 79. 


\section{ARCHIVES DE SOCIOLOGIE DES RELIGIONS}

leur individualité pour ne plus devenir que des moments divers d'un seul et même développement ». Cela ne signifie pas qu'à l'intérieur d'un type ou d'une espèce il ne puisse y avoir un mouvement. L'espèce France connait différentes phases mais demeure ce qu'elle est.

Ces phases sont soit des accidents ou des anomalies (15), soit l'accomplissement du type. C'est donc par la société moderne que le sociologue accède à la société tout court. La société moderne lui donne ce tout de composition (sommation des parties), mais aussi le typique qu'il recherche. Sur ce point, Durkheim se rapproche de Marx pour qui l'anatomie de l'homme permet de comprendre celle du singe.

Traitant de la famille dans un cours de 1888 à la Faculté des Lettres de Bordeaux, il dit (16) : "La famille moderne tient en raccourci tout le développement historique de la famille, où s'il n'est pas juste de dire que tous les types familiaux se retrouvent dans le type actuel parce qu'il n'est pas démontré que tous ont été en communication directe ou indirecte avec lui, du moins cela est-il vrai de beaucoup. Ainsi considérées, les différentes espèces de famille qui se sont successivement formées apparaissent comme les parties, les membres de la famille contemporaine que l'histoire nous offre, pour ainsi dire, naturellement dissociés". Et dans la société moderne " la démographie en effet parvient à exprimer presque au jour le jour les mouvements de la vie collective " (17).

Mais en même temps, du fait même que la continuité est la dimension essentielle de l'histoire, on ne peut en chasser le christianisme. Il y a à la fois une continuité et une discontinuité du christianisme dans l'histoire. Bien plus, il y a une opposition sociale au christianisme. Le christianisme est au centre d'une contradiction (18). Mais je ne puis faire la science de cette continuité et de cette discontinuité, ni de la contradiction, puisque l'espèce sociale constituée ne porte pas en elle la religion sinon comme le besoin d'une foi.

La théorie de la société est donc incomplète. En même temps me font défaut et la théorie de la connaissance, puisque j'exerce une intelligence scientifique sans conscience transcendantale, c'est-à-dire sans la conscience de ses origines, et une praxéologie, puisque je puis montrer la stagnation et du même coup la nécessité de la foi mais que je ne sais comment la créer.

La religion, le christianisme en particulier, ne font pas partie de la vie moderne. Nous avons déjà indiqué qu'il n'y avait pas, à l'inverse du mouvement socialiste, de mouvement chrétien dont je puisse faire un genre. Mais je ne puis pas davantage constituer le christianisme en chose, parce que je me trouve en présence d'une poussière de faits chrétiens, d'une stagnation ambiguë ou d'une causalité qui n'a rien de spécifique. Ainsi le lien familial est plus distendu chez les protestants que chez les catholiques. Mais la variable indépendante, la cause, ce n'est pas la

(15) "Les révolutions au sens propre du mot sont choses aussi impossibles que les miracles ", dans "L'évolution et le rôle de l'enseignement secondaire en France ", Reưe bleue, 5, 1906, p. 70-77.

(16) "Introduction à la sociologie de la famille \#, Annales de la Faculté des Lettres de Bordeaux, 1888, p. 263-64.

(17) Ibid., p. 271.

(18) Discontinuité-Continuité-Contradiction. Ces catégories que nous suivons dans le fil de la pensée durkheimienne, Lévi-Strauss les envisage d'un point de vue logique à la fin de Le Totémisme aujourd'hui, P.U.F., 1962, p. 141. 
religion, c'est la famille (19). A proprement parler je n'ai pas d'accès à la religion. Je pressens un besoin de foi, je sais que tout corps social adhère à une foi, mais je ne puis rien en dire.

\section{II. - LA SOlution : L'Étude SOciologique de la REligion.}

Durkheim indique dans la suite de la lettre que nous citions au début de cet article que son changement d'orientation "était dû tout entier aux études d'histoire religieuse (...) et notamment à la lecture de Robertson Smith et de son école ". Mais il ajoute plus loin que les historiens anglais des religions sont étrangers à l'idée sociologique. Il n'en reste pas moins intéressant de voir ce que Durkheim pouvait prendre chez Robertson Smith. Il y trouve l'affirmation du caractère national de la communauté de langue, de territoire, qui par son biologisme nous éloigne de la notion d'espèce sociale. Mais il y trouve surtout l'idée qu'il faut remonter aux formes simples d'une espèce sociale, plus préciséement aux "germinal principles " (20).

Il affirme en effet dans les Règles qu'il faut partir du simple pour comprendre le complexe. Nous avons vu l'usage que Durkheim fait de cette directive dans l'étude de la famille. Mais l'idée d'un germe initial va plus loin en ce sens qu'il n'y a pas passage du simple au complexe par voie de collection progressive des parties. Ise simple porte déjà en lui le complexe. La partie est déjà elle-même le tout. "Un tout ne peut être défini que par rapport aux parties qui le forment " (21). Ia superstructure religieuse n'est pas un tout au-delà des parties. Chaque partie est la superstructure, chaque individu est la société. "Comme la partie rappelle le tout, elle évoque aussi les sentiments que le tout rappelle " (22).

Une telle idée du tout permet d'expliquer le phénomène de la foi puisqu'il y a fusion entre les parties et le tout, entre les individus et le groupe (23). Elle permet aussi d'expliquer la contradiction et la discontinuité. En effet, dès le germe se manifeste la contradiction entre le sacré et le profane. Il y a une bipartition du monde qui n'explique pas la mort du christianisme mais rend compte de la contradiction. Le germe contradictoire est la forme a priori à partir de laquelle d'autres contradictions sont possibles, en particulier celle qui se manifeste entre l'esprit laïc et l'esprit religieux et que Durkheim situe dès le Moyen Age, à l'intérieur même des institutions universitaires.

Par là même peut être expliquée une lutte historique certes, mais aussi une discontinuité. L'esprit laïc succède à l'esprit religieux. 'Toutefois il faut se garder de voir ici un retour aux séries (montante-descendante) du positivisme. La contradiction n'est pas une synthèse "après coup" de l'Histoire, mais une catégorie.

(19) Nous schématisons à dessein. Selvis a montré ("Durkheim's Suicide and problems of empirical research", American Journal of Sociology, 63 (6), mai 1958, p. 607-19) que la variable indépendante a un statut ambigu chez Durkheim. De fait, à diverses reprises, se trouve évoqué dans Le Suicide le pouvoir d'adhésion suscité par la foi née d'une vie collective intense.

(20) Cette expression se trouve à plusieurs reprises dans Kinship and Marriage in Early Arabia, Cambridge, 1885. Lectures on the Religion of the Semites, Edimbourg, 1889.

(21) Les formes élémentaires de la vie religieuse, Paris, P.U.F., 1869, p. 49.

(22) Ibid., p. 328.

(23) La religion, définie conme une croyance et une règle dans L'Année sociologique (II, 1897-98, p. 128), est définie dans les Formes élémentaires comme la croyance d'une Eglise, c'est-à-dire d'une communauté vivante. 


\section{ARCHIVES DE SOCIOLOGIE DES RELIGIONS}

En même temps la continuité est assurée entre le germe et les formes complexes parce que le germe porte en lui d'autres catégories comme celle de genre, de force, d'espace et de temps. L'espace n'est pas un tout indéfini comme le pensait Kant. C'est une organisation des représentations comme le voulait Hamelin et cette coordination est une fonction sociale. Le germe est donc un tout organisé. Cette organisation porte en elle, sur la base d'une foi commune, d'une existence sociale commune des concepts, des "notions-types" (24), et aussi une contradiction, celle qui existe entre la religion et la science. Cette contradiction, dans la mesure où la science s'attache aux choses, dans la mesure où elle est considérée à certains moments comme une profanation, recoupe en partie la bipartition sacré-profane tout en maintenant une certaine continuité entre la religion et la science. Il s'ensuit que la science succède à la religion, se "substitue " (25) à elle sans pouvoir totalement la remplacer et sans rompre non plus avec elle, puisque les catégories comme le genre, l'espace, etc., sont d'origine religieuse (26).

\section{III. - Portée de cét'te solution.}

Durkheim parle d'une "révélation ». De fait les études sur l'éducation et bien entendu l'énorme travail sur les Formes élémentaires de la vie religieuse vont être commandées par cette théorie du germe.

On peut penser que cette théorie, et par voie de conséquence la solution apportée au problème de l'étude sociologique de la religion, sont abstraites sinon toutes verbales. Il est exact que Durkheim est allé de la philosophie vers la sociologie (27). Plus précisément, à partir de Renouvier, il a constamment médité sur le problème kantien de la synthèse a priori, ou si l'on veut il s'est demandé comment un tout était pensable scientifiquement.

Cette question, dont nous venons de tenter de montrer quelques aspects, est décisive pour la pratique de la recherche sociologique. En effet, pour constituer la science je dois, en un sens, comme l'a vu Kant, déjà posséder la science. Si je ne veux pas me fier aux idoles contenues dans les mots, si je ne veux pas non plus simplement transcrire une poussière de perceptions, il me faut tout au moins une vue du tout de la chose : solidarité de la société moderne - suicide. J'accède à cette vue d'abord par un tri des phénomènes qui me donne une classe, puis par deux voies, l'une qui consiste à suivre la sommation des parties (exemple la solidarité), l'autre qui consiste, soit par une dialectique des thèses, soit par l'étude d'un indicateur (le Droit, les statistiques), soit par les deux moyens réunis (c'est le cas de l'étude sur le suicide), à poser une hypothèse ou plutôt un ensemble causal, un type. Le type est un tout, une synthèse $a$ priori grâce à laquelle la multitude des faits de suicide ou des faits politiques devient intelligible.

La preuve du caractère réel du type vient de l'application de la méthode des variations concomitantes. Ainsi s'amorce un rapport scientifique entre la sociologie et l'histoire. Ce recours reste cependant très limité avant 1895. Du moins l'histoire est-elle réduite à une continuité, dont le fondement tient à la théorie des forces sociales que révèlent les types : sommation démographique - pouvoir

(24) Formes élémentaires, op. cit., p. 624.

(25) Ibid., p. 613.

(26) Ibid. "Les notions essentielles de la logique scientifique sont d'origine religicuse ".

(27) A l'Ecole Normale on le surnommait "le métaphysicien". Voir G. DAvy, "Emile Durkheim ", Reoue de métaphysique et morale, 1019, 1. 
intégrateur du groupe - montée de la démocratie. Mais alors, ni la discontinuité et la contradiction que manifeste le problème de la foi, ni la multiplicité des catégories, ni l'action même ne sont intelligibles. La théorie du germe lie histoire et sociologie en une nouvelle théorie du tout social.

Mais quelle histoire ? quelle sociologie ? Il ne s'agit ni de l'histoire à la mode spencerienne qui prend " un aspect sériaire et simple " (28), ni de celle de Robertson Smith, amalgame non moins confus. Il s'agit d'une histoire rapprochée de l'ethnographie, fondant cette dernière en même temps qu'elle en reçoit des bases plus assurées. "C'est seulement en rapprochant les faits ethnographiques des faits historiques qu'on peut dépouiller les premiers de leur ambiguïté. De ce que l'on constate une pratique dans un certain nombre de tribus arriérées, ou qui passent pour telles, on n'a pas le droit de conclure qu'elle est primitive. Mais il n'en est plus de même si on la retrouve à la base des pratiques similaires qui se sont successivement constituées au cours de l'histoire. On est vraiment assuré qu'elle est première en date, si l'on peut montrer que tout ce qui a suivi en est dérivé. C'est l'enchaînement causal des faits qui manifeste le mieux leur ordre de succession; or, pour l'établir, il faut sortir des sociétés inférieures et de l'ethnographie" (29). Ce passage, quoiqu'il fasse mention d'une succession causale, est encore très proche du texte de 1888 sur la famille. Fn fait l'histoire et la sociologie sont fondées et unies dans une nouvelle idée du tout, du type. Le terme "opinion " exprime le mieux cette nouvelle idée (30). L'opinion est une norme, une superstructure (31). Mais aussi un consensus, et encore le moule des catégories et de la contradiction.

L'opinion est en même temps vécue comme adhésion, exaltation et discipline Elle est en un sens intériorité. C'est pourquoi, lorsqu'il aborde l'institution scolaire, Durkheim dit qu'il faut la connaître "non du dehors mais $d u$ dedans, c'est-à-dire par l'histoire" (32).

Ainsi une histoire sociologique est possible parce que la réalité sociale est à la fois typique, temporelle par son déroulement discontinu ou contradictoire, et vécue. Une sociologie est possible à partir de l'opinion, indicateur majeur, parce que l'ensemble des variables d'une espèce sociale peut être dégagé grâce à l'histoire.

\section{IV. - Critiques.}

La solution dont nous venons d'indiquer les principaux traits n'est évidemment pas sans failles. Lévi-Strauss dans son ouvrage sur le totémisme, marque - en plus du caractère artificiel de la théorie du totémisme - la contradiction entre l'origine affective de la foi, de l'opinion, et son origine intellectuelle. Il privilégie Bergson par rapport à Durkheim en montrant que l'intellectualisme durkheimien a été moins apte que l'intuition bergsonnienne à saisir le sens de l'intelligence primitive. Il nous semble toutefois que, sans être tout à fait levée, cette contradiction est moins globale que ne l'indique Lévi-Strauss, pour peu que l'on se réfère à la théorie durkheimienne du comportement. Comme ses contemporains, Durkheim donne une importance considérable au problème de l'habi-

(28) Les Règles, p. 20.

(29) "L'origine du mariage d'après Westcrmarck. Revue critique ", Revue de métaphysique et de morale, déc. 1895 , p. 606-23 (p. 606).

(30) Formes élémentaires, op. cit., p. 626.

(31) Ibid., p. 328.

(32) Education et sociologie, Paris, Alcan, 1922, ch. IV. "L'évolution et le rôle de l'enseignement secondaire en France ", art. cit., p. 157. 
tude (33). Lorsqu'il se demande dans son cours de 1888 sur la famille comment atteindre en elle-même la structure d'un type familial, il dit qu'on la trouve "dans ses manières d'agir consolidées par l'usage qu'on appelle les coutumes, le droit, les mœurs " (34).

L'habitude n'est pas tant définie par sa fréquence que par sa "vertu impérative ". C'est pourquoi, dans Les Formes élémentaires de la vie religieuse, l'habitude se trouve complétée sinon dépassée par l'exemple. "On objectera que souvent, chez l'individu, par le seul effet de la répétition, des manières d'agir ou de penser se fixent et se cristallisent sous forme d'habitudes qui résistent au changement. Mais l'habitude n'est qu'une tendance à répéter automatiquement un acte ou une idée, toutes les fois que les mêmes circonstances la réveillent; elle n'implique pas que l'idée ou l'acte soient constitués à l'état de types exemplaires, proposés ou imposés à l'esprit ou à la volonté. C'est seulement quand un type de ce genre est préétabli, c'est-à-dire quand une règle, une norme est instituée que l'action sociale peut et doit être présumée» (35).

L'emblème peut être rangé aux côtés de l'exemple. La foi, la conduite sont donc tributaires de l'emblème ou de l'exemple. Plus fondamentalement, l'apprentissage lui-même n'est pas possible sans l'exemple. L'essentiel de la pédagogie durkheimienne est dans l'exemple, dont l'efficace tient à la réceptivité de l'enfant à la suggestion (36).

Ainsi s'établissent des ponts entre la valeur et l'acte, la croyance et le rite. De même la catégorie est proche de l'outil (37). Certes la tendance à l'idéalisme est indéniable (38). Il est vrai aussi qu'il y a contradiction entre l'affectivité et le caractère intellectuel de la catégorie, mais Durkheim a cherché à relier les différents éléments de l'opinion dans une théorie plus raffinée de l'habitude. D'autre part, dans la mesure où la famille est le lieu où naît l'habitude, l'initiation de l'exemple, on comprend mieux encore comment peuvent se lier existentiellement l'affectivité, l'habitude et, au-delà, les catégories (39).

En ce sens il nous semble exagéré de situer, comme le fait Lévi-Strauss, Bergson plus haut que Durkheim. Bergson met en place (40) abstraitement les catégories. Durkheim chercher à saisir dans le concret les différents milieux et modes de développement des croyances, des catégories et des rites.

On peut aussi s'interroger sur le caractère scientifique du concept de germe, de la nouvelle idée du tout que l'on découvre dans les Formes élémentaires et dans

(33) "L'irrésistible autorité de l'habitude ", Revue Philosophique, 1886. "Ce qui fait la force et l'autorité de toute discipline c'est l'habitude ".

(34) Cours cité, p. 267.

(35) Formes élémentaires, op. cit., note de la p. 620.

(36) L'autorité du maître vient du for intérieur. "Le sentiment qu'il éprouve, il le suggère aux élèves par la parole, par le geste, par l'exemple ». Education morale, op. cit., p. 199. "L'enfant imite parce que sa conscience naissante n'a pas encore d'affinités électives bien marquées et par suite elle s'assimile sans résistance et sans peine à toutes les impressions qui lui viennent du dehors ". Ibid., p. 252.

(37) Formes élémentaires, op. cit., p. 27 : "entre les trois notions d'outil, de catégorie et d'instituts il y a une étroite parenté".

(38) Voir G. Davy, "Emile Durkheim ", art. cit., 1920, p. 102.

(39) G. DAvy, art. cit., p. 198 : "La famille a peut-être été un sujet d'étude et de cours de prédilection. Il voyait en elle le foyer moral par excellence *. Progressivement, Durkheim ne rédige plus dans l'Année sociologique que la rubrique de la famille.

(40) Voir l'apologue de Péguy in Henri Bergson. Essais et témoignages (recueillis par Albert Béguin et Pierre 'Thévenaz), Neuchâtel, La Baconnière, 1943. Un témoignage inédit de Péguy : "D'autres avaient combattu la sociologie, la psycho-physiologie, la pédagogie : les Bergsoniens s'étaient contentés de l'estimer" (p. 14). 
les travaux ultérieurs. On peut dire qu'elle est commandée par l'objet d'étude, la religion. Est-elle pour autant scientifique ? N'est-elle pas idéologique au sens où l'entend Althusser (41) ? Durkheim, dont nous avons vu à quel point il tendait vers un concept organisé, admet cependant que sa théorie ne peut pas ne pas être idéologique. "Sans doute, dit-il, on peut prendre l'opinion comme objet d'étude et en faire la science; c'est en cela que consiste principalement la sociologie. Mais la science de l'opinion ne fait pas l'opinion; elle ne peut que l'éclairer, la rendre plus consciente de soi. Par là, il est vrai, elle peut l'amener à changer; mais la science continue à dépendre de l'opinion au moment où elle paraît lui faire la loi ; car comme nous l'avons montré, c'est de l'opinion qu'elle tient la force nécessaire pour agir sur l'opinion" (42).

Dans ce cas, le problème de la nature idéologique ou scientifique du concept de germe devient le problème du substrat social, de la terre originaire où s'enracine la théorie durkheimienne de la religion.

\section{V. - Durkheim Rendu a SA terre. - Quelques hy pothèses.}

Nous pouvons avoir une idée de ce problème parce que Durkheim apparaît comme le morceau d'un passé donné et comme l'auteur d'une œuvre relativement close ; en même temps, ce passé n'est pas complètement à l'extérieur de nous. Il est nôtre dans notre activité de sociologue. Plus précisément, comment se fait-il que, dans une aire culturelle donnée (Angleterre, Allemagne, Italie, France), à la fin du $19^{\mathrm{e}}$ siècle, on puisse dire "Dieu est mort ", alors que rien n'est pensable sans l'histoire qui porte en elle le christianisme, comment peut-on lier continuité et discontinuité de la religion, religion et non-religion, et trouver dans ce problème le moyen de fonder la sociologie telle que nous la pratiquons aujourd'hui?

Dans le cas de Durkheim, les voies à explorer nous semblent être les suivantes :

1) "Petit" juif, Durkheim vit l'expérience de la différence (43) sinon de la haine et du mépris, puis le problème du dépassement de cette condition de telle manière que seule une connaissance totale de la France en ses racines lui permette d'accomplir ce dépassement. C'est là chez lui, comme chez Weber en un autre sens, une des sources de sa soif de réalité (Wirklichkeitssättigung) (44).

2) S'il est vrai que la seule société que je puisse penser est la France, s'il est vrai qu'un sociologue ne peut dire que son propre sol, il est non moins vrai, à ce moment du $19^{\mathrm{e}}$ siècle, que la France n'est pas pensable sans l'Allemagne. Pour Durkheim il ne s'agit ni d'y trouver la dialectique, comme le tente Jaurès dans sa thèse complémentaire, ni de définir une doctrine socialiste par voie de comparaison comme le fait Lucien Herr. Durkheim n'est pas assez politique, pas assez

(41) Louis Althusser, "Esquisse du concept d'histoire ", La Pensée, no 121, juin 1965, p. 3-21. Althusser oppose le tout spirituel leibnizien au tout marxiste. Le premier implique une co-présence, une expression du tout dans chaque partie. "Tous les éléments du tout sont donnés dans une co-présence qui est elle-même la présence immédiate de leur essence devenue ainsi immédiatement lisible en eux ", p. 6. Ce tout est en fait le produit immédiat du langage et de la pratique, phénomène et théorie confusément assemblés. Il est idéologique en ce sens qu'il méconnaît, masque la réalité sans pouvoir faire autrement que la masquer. Seule la construction d'un concept, d'un tout complexe et hiérarchisé donne la réalité.

(42) Formes élémentaires, op. cit., p. 626. $424 \mathrm{p}$.

(43) Pierre Aubery, Milieux juifs de la France contemporaine, Paris, Plon, 2e éd. 1962,

(44) Fleischmann, "De Weber à Nietzsche ", Archives européennes de Sociologie, V, 1964, 2, p. $190-238$ (p. 194). 
français et demeure trop déchiré pour que ces chemins lui soient praticables. L'Allemagne c'est pour lui cet "état d'innocence " que constatait Lucien Herr, ce corps social homogène de fait que la France n'a pas encore réalisé, qu'elle va réaliser par le socialisme, mais qu'elle est la seule à pouvoir penser (45).

3) Penser radicalement le corps social implique une physique sociale, c'est-àdire que la totalité est atteinte par l'intermédiaire des parties soit comme une sommation, soit comme la constitution de types liant entre elles deux ou trois variables. L'origine du tout (son fondement transcendental) est dans une vision ordonnée de la société moderne. Cela signifie que l'histoire n'est pas un récit ni même une suite de séries, mais l'ébauche, la réplique inchoative du présent en même temps que le présent est encore le passé. La solidarité mécanique est dans le présent en même temps que la solidarité organique.

4) Cependant le présent porte en lui une discontinuité essentielle et une contradiction, toutes deux nées du destin du christianisme et de la vie politique européenne. L'intellectuel, qui par essence se consacre tout entier à l'intelligence (46), trouve dans la science de l'opinion un nouvel accès au tout et donne le sens de la discontinuité et de la contradiction dans une nouvelle sociologie, liée à l'histoire, grâce au concept de germe.

5) Est-ce à dire que la physique sociale soit abandonnée ? En aucun cas. Le problème de la constitution de la sociologie devient : comment une logique de la société qui soit en même temps le langage d'un parti est-elle possible ? Par la médiation du concept de germe, la physique sociale devient physique des milieux et psychologie collective : l'Université - l'école (" milieu social organisé ") la famille. Le milieu lie les différentes variables, en particulier celles qu'on appelle valeurs, normes. C'est sur cette base théorique qu'il convient d'apprécier l'action de Durkheim dénoncée (et déformée) par la droite, érigée en problème de l'histoire de la sociologie par Lazarsfeld (47). Il serait également possible de reconsidérer le courant qui va de Durkheim à Lazarsfeld à l'aide du concept de milieu.

6) De même, l'étude comparative de la naissance des grands sociologues de la fin du $19^{\mathrm{e}}$ siècle nous semble devoir gagner à s'établir sur ce même concept de milieu.

En gros, il faut entendre par étude selon les milieux, une investigation portant sur les variables écologiques, démographiques, institutionnelles et idéologiques, historiquement situées, d'un groupement social producteur de valeurs (48).

7) Il serait tout aussi utile de tenter d'expliquer l'attitude faite de réserve et d'admiration mêlées, que l'on constate chez les sociologues qui ont suivi Durkheim. Ils ont coutume de séparer la méthode du prophétisme, la sociologie du sociologisme. Cette distinction relative pourrait s'expliquer à la fois par le

(45) Cf. la formule de R. Aron: Durkheim, "contre-partie sociologique du socialisme ", in "Sociologie et Socialisme ", Annales de l'Université de Paris, 1960. Bien entendu, ce socialisme serait à définir par une étude attentive des rapports de Durkheim avec Jaurès, Herr, et une interprétation de la critique de Sorel, Devenir social, I, avril 1895.

(46) Voir la définition de l'intellectuel in "L'individualisme et les intellectuels ", Revue bleue, 2 juill. 1898. (Il s'agit de l'affaire Dreyfus).

(47) Paul Lazarsfend, "Notes on the History of Quantification in Sociology. Trends, sources and problems", in Harry Woolf, éd., Quantification. A History of the meaning of measurement in the naturel and social sciences, N.Y., The Bobbs-Merrill Company, 1961, p. 147-203.

(48) L'étude de Fleischmann précédemment citée est un premier pas dans ce sens. On peut trouver aussi certaines suggestions in J. BEN-DAvid et A. Zloczower, "Universities and Academic Systems in modern Societies ", Archives europeénnes de Sociologie, III, 1962, 1, p. 4584. (Tout ce qui concerne les choix possibles dans l'Université allemande). 
fait que le socialisme, l'Université, l'école, la famille auraient changé, et par le fait que le même rationalisme, la même volonté radicale de s'en tenir aux phénomènes, la même tendance à aboutir à un concept construit, seraient encore à la base de la sociologie.

Nous pourrions à notre tour opérer la même distinction avec des attendus différents. (Une nouvelle Université - Une nouvelle idće de la famille, etc.).

Mais peut-on ainsi désarticuler la pensée durkheimienne ? Durkheim s'accomplit scientifiquement dans sa théorie de la religion. Il n'ajoute pas un prophétisme à une méthodologie. Du moins c'est ce qu'il croit et déclare. Peut-être faut-il prendre cette thèse au sérieux ?

Nous avons montré que la théorie durkheimienne du tout consistait en un concept hiérarchisé et en même temps porteur des diverses catégories et de la contradiction. Ce concept est empiriquement incomplet en ce sens qu'il ignore pratiquement la paysannerie et la classe ouvrière. Cette insuffisance empirique entraîne une insuffisance théorique, une hićrarchie idéologique. Le tout est certes organisé mais dominé par une idée globale de l'opinion. Le tout se manifeste dans l'emblème, l'exemple, sans que nous puissions sinon analyser du moins lire, entendre cette manifestation. De même, l'histoire est faite de la simple "substitution" des emblèmes les uns aux autres (le prêtre, l'intellectuel, le professeur). Dans ce cas peut-on être encore durkheimien, même d'un point de vue uniquement méthodologique? Par exemple, quelles que soient les vertus heuristiques du concept de milieu, il demeure une première approche, utile mais trompeuse, si elle ne s'adosse pas à une combinatoire des milieux, une théorie des fonctions sociales possibles dans une espèce sociale donnée. L'origine de ce caractère idéologique de la pensée durkheimienne nous semble devoir être recherchée dans la compréhension d'un certain nationalisme. La volonté d'intégration à la France a, en définitive, masqué, oblitéré la complexité de la France et par voie de conséquence, relle de la société industrielle.

\author{
Joseph SumpF \\ Centre d'Etudes Sociologiques \\ (C.N.R.S.)
}

